\title{
Reconstrucción conceptual del sistema de repitencia en Educación Básica: Una propuesta de aprendizaje mediado basada en el Cambio Generativo
}

\author{
Conceptual reconstruction of the repetition system in elementary education: \\ A proposal of mediated learning based on Generative Change
}

\section{Elizabeth Martínez}

Instituto de Ciencias de la Educación

Facultad de Filosofía y Humanidades

Universidad Austral de Chile

elymartinezpalma@hotmail.com

Telf.: (56) 63221262

\section{RESUMEN}

Este trabajo propone reconceptualizar la noción de repitencia, como indicador único de evaluación del rendimiento escolar, centrada en la medida psicométrica de la inteligencia y en la evaluación subjetiva, basada en este parámetro, que los educadores establecen para niños de cuarto año básico. Esta reconstrucción conceptual postula una relación compleja y dinámica entre aprendizaje e inteligencia, considerando capacidades, procesos y estructuras para la potenciación del desarrollo intersubjetivo manifiesto en la formación del estudiante. El análisis se centra en la estructura operacional del aprendizaje mediado, como centro orientador del desarrollo individual, y propone indicadores que dan cuenta de la conducta inteligente como expresión de la experiencia social y cultural del niño. De este modo, visibiliza las relaciones que emergen entre aprendizaje y desarrollo en el contexto del cambio generativo.

Palabras clave: proceso de aprendizaje, desarrollo infantil, inteligencia, cambio generativo en DBM®.

\begin{abstract}
This paper proposes to reconceptualize the notion of repetition, as the sole indicator of school performance evaluation, focused on psychometric intelligence measurement and subjective assessment based on this parameter that educators set for fourth grade children. This conceptual reconstruction postulates a complex and dynamic relationship between learning and intelligence, considering capabilities, processes and structures for the enhancement of inter-development evident in student training. The focus of analysis centers on the operational structure of mediated learning as guiding individual development, through new indicators to show the intelligent behavior as an expression of social and cultural experience of the child, to visualize the relationships that emerge between learning and development in the context of generative change.
\end{abstract}

Key words: learning experience, child development, intelligence, generative change in DBM®. 


\section{INTRODUCCIÓN}

Sobre la base de los resultados evidenciados en el trabajo titulado "La Repetición de cursos en Educación Básica: Estudio Evaluativo del Sistema en alumnos con distinto número de repitencias" (Bravo, 1976), es posible advertir la relación lineal manifiesta entre las nociones de inteligencia, capacidad de aprendizaje y rendimiento escolar, que el estudio da a conocer en su análisis. Esta relación remite a la convicción tradicional que educadores, padres y sistemas educativos han construido a partir de las influencias y aportaciones de algunas aproximaciones clásicas que han caracterizado la inteligencia como factor preponderante en el aprendizaje y rendimiento escolar. Se trata de una concepción dominante hasta los años sesenta, e influyente aún en la actualidad (Coll y Onrubia, 2001).

De acuerdo con la investigación en cuestión, y según antecedentes del sistema de promoción escolar en Chile entre los años 1972 y 1976' "el número de repitencias, del grupo en estudio ${ }^{2}$, guarda relación con la edad, con la inteligencia según los tests y con la evaluación subjetiva de los maestros de la inteligencia de los alumnos" (Bravo, 1976: 16). Esta relación entre variables sostiene que la inteligencia, medida en base a indicadores psicométricos, es la causa de la repitencia previa de cursos en niños que se encuentran en $4^{\circ}$ Básico. Además, la investigación da a conocer la alta incidencia que la evaluación de los maestros tiene para la promoción de los estudiantes (en el contexto donde se lleva a cabo la investigación). En este caso, se advierte que "estos resultados parecen indicarnos que esta evaluación se hiciera principalmente en base al juicio que se forman los maestros de la inteligencia del niño, y no en base al rendimiento logrado en las materias escolares" (Bravo, 1976: 16). Por tanto, la correlación entre las variables inteligencia y la evaluación subjetiva de los maestros sobre ella, codificada en el rendimiento intelectual, constituye el elemento de mayor peso en la promoción escolar de los estudiantes.

De manera particular, el análisis y discusión de los resultados permite a los investigadores cuestionar el "proceso de evaluación - promoción y repetición" utilizado en el contexto histórico-educativo en donde se aplica este sistema, según el sector investigado. En este sentido, los resultados remiten el rendimiento escolar a las capacidades intelectuales de los niños y evidencian que este rendimiento no manifiesta diferencias significativas entre estudiantes que repitieron los cursos en varias oportunidades y entre aquellos que fueron promovidos sin repitencia. De este modo, tanto la promoción automática como la repitencia han sido puestos en tela de juicio por las evidencias investigativas del estudio (Bravo, 1976).

Este cuestionamiento permite dar luces respecto a un salto importante en materia de sistematización de procesos educativos a nivel escolar en el periodo que se realizó la investigación. Tal como lo menciona el autor, la promoción automática y la repetición de cursos no pueden considerarse como una solución pedagógica que logre mejorar el rendimiento escolar. De manera conjunta, en sus enunciados declara que no se establece como criterio único de evaluación del rendimiento escolar "la cantidad de materia asimilada", en este caso por el estudiante, como una "asimilación automática de conocimientos" (Bravo,

Período en que se llevó a cabo la investigación.

"La muestra del estudio está constituida por todos los escolares repitentes del $4^{\circ}$ año básico de 22 escuelas del $6^{\circ}$ Sector Escolar de Santiago (Ñuñoa, La Florida, La Reina). El número de repitentes alcanzó a 175. Se incluye, además, un grupo elegido al azar de no repitentes de condiciones escolares similares (igual escuelas y curso, e igual proporción de sexos)" (Bravo, 1976: 9). 
1976:14). Los investigadores, además, mencionan que no conciben al escolar "como una caja que haya que llenarse de conocimientos, ni como una pequeña calculadora que se perfeccione en la medida en que tenga capacidad para más operaciones y más memoria" (Bravo, 1976: 14).

Sin embargo, y a pesar de los nuevos enfoques que se vislumbran (semánticamente acerca del aprendizaje), en las premisas manifiestas por el autor, el rendimiento del estudiante queda supeditado a las capacidades intelectuales evidenciadas en términos cuantitativos y ligados a parámetros basados en técnicas matemáticas de correlación, como el análisis factorial, para obtener información sobre la estructura de estas capacidades (Coll y Onrubia, 2001). Es por tanto que el interés de nuestro artículo se centra en repensar la estructura operacional del aprendizaje, a través de nuevos indicadores que den cuenta de la conducta inteligente como expresión de la experiencia social y cultural del niño, para visibilizar las relaciones que emergen entre aprendizaje y desarrollo.

Como objetivo, nuestro trabajo propone reconceptualizar la noción de repitencia como indicador único de evaluación del rendimiento escolar, centrado en la medida psicométrica de la inteligencia, y la evaluación subjetiva basada en este parámetro, que los educadores establecen para niños de cuarto año básico. En esta reconstrucción conceptual, se propone focalizar el aprendizaje mediado como centro orientador del desarrollo individual, a partir de la experimentación del cambio generativo manifiesto en el contexto situacional en donde cobra sentido la experiencia vital del infante. De acuerdo con este objetivo, se postula una relación compleja y dinámica entre aprendizaje e inteligencia considerando la multiplicidad de capacidades, procesos y estructuras, a nivel individual, social y cultural, implicadas en el comportamiento inteligente para la potenciación del desarrollo intersubjetivo que construye el estudiante en su proceso de formación.

La propuesta se organiza en dos apartados que dan cuenta de las orientaciones que explicitan el objetivo del trabajo. El primer apartado muestra aproximaciones teóricas provenientes de la psicología que caracterizan el desarrollo infantil y la estructuración de la inteligencia. El segundo apartado presenta un modelo metodológico transversal de aprendizaje, basado en el DBM ${ }^{3}$, que amplía las posibilidades de desarrollo a partir del cambio generativo.

\section{APROXIMACIONES TEÓRICAS SOBRE EL DESARROLLO INFANTIL Y LA INTELIGENCIA}

Establecer una relación entre las aproximaciones teóricas que han estudiado el desarrollo infantil y la inteligencia, remite a una concepción genético-cognitiva del desarrollo y del aprendizaje. Esta concepción permite evidenciar que "el nivel de competencia intelectual de una persona en un momento determinado de su desarrollo depende de la naturaleza de sus esquemas, del número de los mismos y de la manera en que se combinan y coordinan entre sî" (Coll y Martí, 2001:68). En estos enunciados se advierte uno de los postulados presentados en los estudios de Piaget $(1961,1973)$ y de sus colaboradores. Piaget, en su posición interaccionista, en oposición a la tesis empirista de los enfoques de la época, establece que en cualquier aprendizaje la intervención del sujeto y de los objetos está

Developmental Behavioural Modelling o Modelo Conductual Desarrollativo, diseñado por John McWhirter. 
organizada por factores internos de equilibración en el individuo, específicamente en el aprendizaje de estructuras lógicas. Es por tanto que, para el autor, existe una íntima conexión entre aprendizaje y desarrollo del pensamiento en el niño. Este desarrollo se organiza en estadios o niveles que determinan las habilidades cognitivas del sujeto y, a través de ellas, miden su aprendizaje ${ }^{4}$.

La teoría sociocultural, por su parte, planteada por Lev Vygotski (1979), manifiesta la relación bidireccional entre el desarrollo cognitivo del individuo y el contexto sociocultural de pertenencia. Para Vygotski, según lo plantea Shaffer (2007), el niño desarrolla sus capacidades mentales para resolver problemas e interpretar el entorno de acuerdo con las normas y valores inscritos en la cultura. En este sentido, la cognición humana "es intrínsecamente sociocultural: la afectan las creencias, los valores y las herramientas de la adaptación intelectual transmitida a los individuos por su cultura" (Shaffer, 2007: 274). Esta teoría cuenta hoy en día con un extenso cuerpo de investigaciones desde diversos ángulos (Psicología evolutiva y Psicología de la Educación) que han aportado a la relación desarrollo y aprendizaje, identificando a la escuela (institución educativa) como un espacio cultural en donde se producen estos aprendizajes (Cubero y Luque, 2001).

Desde una óptica distinta, las teorías del procesamiento de la información ${ }^{5}$, influenciadas por los sistemas informáticos, se ocupan de la secuencia y ejecución de los procesos cognoscitivos para entender el funcionamiento de la mente humana (Schunk, 1997; Shaffer, 2007). Es por tanto que, como corrientes disciplinares, han sido consideradas dentro de las aproximaciones teóricas vinculadas más a la inteligencia y al aprendizaje que al desarrollo. Entre sus precursores es posible identificar a Ebbinghaus (1913), que en sus investigaciones sobre el Aprendizaje Verbal desarrolló contribuciones notables a esta disciplina. También se han considerado los influjos de los principios y supuestos provenientes de la Psicología de la Gestalt, la teoría de la interferencia (Postman 1961), el modelo de dos almacenajes (Craik y Tulving, 1975; Morris, Bransford y Franks, 1977; Moskovitch y Craik, 1976; Nelson 1977), los aportes del modelo multialmacén del sistema procesador de la información que organiza de manera gráfica la forma en que funciona el pensamiento de Atkinson y Richard Shiffrin (1968), entre otros (Shaffer, 2007; Schunk, 2012). De acuerdo con Schunk (1997), los postulados de este cuerpo de teorías han sido incorporados a los estudios del aprendizaje, la memoria, la resolución de problemas, la percepción vidual y auditiva, el desarrollo cognoscitivo y la inteligencia artificial.

Las distinciones expuestas, que se inscriben dentro de un vasto y extenso cuerpo de investigaciones y postulados teóricos, delimitan las posibles congruencias entre desarrollo y capacidades cognitivas, las que aluden a las funciones psicológicas superiores codificadas a través de la inteligencia. Sin embargo, este trabajo plantea un recorrido más amplio de los enfoques que han estudiado el desarrollo infantil con la finalidad de delimitar aproximaciones que evidencian nociones articuladoras entre estos planteamientos y los enfoques que han caracterizado a la inteligencia. En este sentido, el empirismo (representado en la relación estímulo-respuesta), que sucedió al psicoanálisis en las primeras esferas del estudio del infante, no establece relaciones directas con la génesis de los postulados de la inteligencia en su dimensión psicométrica. Sin embargo, las corrientes posteriores van otorgando sentido al proceso de interiorización y apropiación (asimilación,

Este aspecto se describirá en sub-apartado 2.1.

Noción que acuña los postulados de variadas corrientes teóricas del desarrollo cognitivo. 
acomodación y equilibrio para Piaget; zona del desarrollo próximo para Vygotski) de las funciones psicológicas superiores (como estructuras cognitivas) en contextos culturales de interacción, dando sentido a los modelos psicosociales, ecológicos y organicistas en la teorización del desarrollo del niño. De igual modo, los estudios sobre los factores que miden la inteligencia han ido posicionando una visión intersubjetiva de la misma, en donde la cognición se sitúa en espacios socio-culturales y resalta el proceso de interacción en el comportamiento inteligente, como lo evidencia la caracterización de la inteligencia distribuida, por ejemplo.

Más allá de establecer comparaciones o clasificaciones entre un presupuesto y otro, el análisis pretende dilucidar los factores que, situados en la enseñanza, mejoran y optimizan las capacidades intelectuales y de aprendizajes de los niños para fortalecer sus procesos formativos en función de su desarrollo. Estos factores han sido influenciados por las corrientes que a continuación se presentan.

\subsection{ENFOQUES SOCIOCOGNITIVOS Y PSICOSOCIALES EN EL ESTUDIO DEL DESARROLLO INFANTIL}

Una de las bases fundamentales, en el ámbito de la infancia, la constituyen los estudios del desarrollo del sujeto a un nivel psíquico cuya tradición teórica cruza los enfoques psicodinámicos, organicistas y de las leyes de aprendizaje representados en el psicoanálisis, el conductismo, las corrientes cognitivas, cognitivo sociales - evolutivas y los modelos ecológico - sistémicos - comprensivos (Bandura, 1986; Piaget, 1961, 1965, 1973); Bowlby, 1969, 1988, 1993; Rogers,1984; Bronfenbrenner,1993, 1995, 2005; Erikson, 1993; Bruner,1964; Amar y Abello, 1998; Palladino, 2009; Cantón, Cortés et al, 2011).

Estas tradiciones han denotado acercamientos distintivos al estudio del desarrollo infantil, cuyas aportaciones se concentran en referentes específicos, dinámicos y, en algunos casos, enlazados. En primer lugar, desde una perspectiva individual, los estudios del conductismo intentan establecer explicaciones comprensivas acerca de la conducta analizando los modeladores y condicionantes que refuerzan, posibilitan o inhiben el desarrollo de determinados patrones conductuales. Entre sus máximos representantes es posible reconocer a Watson (1913), Skinner (1953), en el estudio de adultos; y Gesell (1933), con sus contribuciones al desarrollo de la conducta infantil, entre otros (Papalia, 1988; Palladino, 2009).

Desde las orientaciones psicoanalíticas, con algunos destacados como Sigmund Freud (1930, 1933, 1960), Melanie Klein (1932), John Bowlby (1969, 1985, 1988, 1993), Donald Woods Winnicott, se perfilan los desarrollos psicológicos conscientes e inconscientes frente a las necesidades, intereses e impulsos de los individuos, cuyos trabajos más recientes denotan la constitución de la subjetividad del niño durante la infancia (Palladino, 2009). Emergiendo en el psicoanálisis, Erikson (1993) en su teoría psicosocial, situada en el Ciclo Vital, posiciona el estudio de la formación identitaria en una relación dinámica, correlacional e influyente entre el desarrollo individual y el contexto social. La formación de la identidad, según lo plantea el autor en su teoría del ciclo vital (Erikson, 1982), establece el desarrollo de tareas psicosociales que el individuo afronta durante su proceso evolutivo, que fortalecerán o dificultarán manifestaciones de seguridad, autonomía, iniciativa y laboriosidad. Estas tareas se materializan en el desarrollo de experiencias vitales en la interacción social del día a día (Guiñez y Martínez, 2015). 
De acuerdo con esta teoría cada individuo debe afrontar ocho crisis o dilemas para desarrollar una identidad completa y estable. Cada crisis implica resolver tareas psicosociales específicas. Las primeras cuatro tareas corresponden a la preparación y construcción de la identidad proyectada hacia la edad juvenil, despertando el accionar autónomo, independiente y responsable ante el modelo de vida a seguir. En esta noción de construcción, el individuo debe generar estrategias de afrontamiento que van a solidificar los estilos que conforman su proceso de desarrollo y madurez frente a las transiciones vitales. El eje central es conocerse a sí mismo, formar la propia individualidad y, a partir de ésta, proyectarse al mundo.

Por su parte el legado cognitivo-evolutivo de Piaget (del cual ya se ha hecho mención) fundamenta el desarrollo de modelos representacionales en la estructuración del pensamiento y la inteligencia, como procesos internos que se despliegan en el mundo exterior de manera adaptativa a través de la asimilación, acomodación y equilibración de esquemas mentales (Beard, 1971; Piaget, 1954,1973). Su trabajo, entre muchos otros, entrega las bases de un modelo constructivista y confluye con las aportaciones de los estudios del aprendizaje y las estructuras de las funciones psicológicas superiores representados por Gagné (1979, 1985), Ausubel (1963), Bruner (1964,1983, 1985), entre muchos otros (Schunk, 1997, 2012). Para Vygotski (1979), según lo expuesto al inicio de este apartado, el concepto de estructuras se consolida en dos aspectos, entendiéndose el primero como punto de partida de todo proceso agenciado en el desarrollo cultural del niño, y el segundo, constituido en el desarrollo cultural, más amplio, el cual canaliza un cambio de la estructura inicial hacia nuevas estructuras. En este sentido, afirma que entre la conducta dirigida al estímulo y la reacción del sujeto se establece un acto mediador que articula el proceso (Palladino, 2009).

Desde una perspectiva organicista, la conducta, aprendida a través de los modelos sociales y el aprendizaje vicario, va constituyendo patrones adaptativos de acuerdo a los referentes vinculares cercanos al niño. Si bien los procesos de socialización son desarrollados por cada uno de los enfoques aludidos, desde la particularidad de su prisma investigativo, es Albert Bandura (1986, 1989, 1991) quien entregaría en los años sesenta una propuesta teórica centrada en el aprendizaje social como base de la imitación de los modelos adultos por parte del niño. Particularmente, Kohlberg (1992), conjuntamente con Piaget (1965), centra parte de su trabajo cognitivo en el desarrollo de la conducta prosocial considerando que el razonamiento moral se basa en la estructura cognitiva y no en el aprendizaje de reglas (morales) de nivel concreto y abstracto. El autor fundamenta que, según estos niveles de razonamiento, el niño va construyendo una moralidad posicionada en los modelos adultos en los primeros años de vida, y que esta moral se diferencia camino a la adolescencia al alero de patrones autónomos (Cantón, Cortés et al 2011).

Las investigaciones más recientes, surgidas a finales del siglo XX aproximadamente, convergen con ciencias emergentes, como las neurociencias, las ciencias cognitivas (teorías del procesamiento de la información) y la influencia de los estudios pospositivistas, otorgando nuevas orientaciones al desarrollo de la conducta humana. La herencia constructivista constituye un legado importante para sus desarrollos teóricos, y establece que los modelos representacionales que construye cada sujeto en particular, acerca de sí mismo y de su entorno, corresponden a procesos relacionales interdependientes que lo sitúan dentro de un engranaje de sistemas. La experiencia no es del todo el mecanismo central en el intercambio con el mundo objetal, natural, social y cultural, como lo fue en las bases iniciales del constructivismo, puesto que, bajo las premisas de estas corrientes, la 
experiencia se entiende como un proceso mediador y canalizador de significados. En este proceso subyacen tres esferas manifiestas en constante movilidad, considerando, como una de ellas, la delimitación de sentidos subjetivos modelados por los individuos de acuerdo a una realidad semiótica constituida en la sociedad y en la cultura. En esta construcción, como segunda esfera, se establecen los consensos que organizan los mecanismos articuladores de los filtros perceptivos que habitúan las rutas atencionales y clasificatorias de las estructuras del mundo de la vida. Y, como tercer aspecto, la relacionalidad en sí misma que conecta, organiza y da forma a estas interacciones en el proceso de construcción de la realidad (Douglas, 1973; Berger y Luckmann, 1986; Amar y Abello 1998; Schunk 2012).

Entre las corrientes sistémico-interaccionistas, se encuentra el original aporte de Bronfenbrenner $(1979,1993,1995)$, cuyo enfoque ecológico ha considerado el carácter fenomenológico de la experiencia humana y, como teoría, comprende el estudio científico de "la progresiva acomodación mutua entre un ser activo en desarrollo, y las propiedades cambiantes de los entornos inmediatos en que vive el sujeto" (Palladino, 2009: 25), de tal modo que las interacciones son afectadas en contextos más amplios en donde se insertan tales entornos.

Otras propuestas se han centrado en el desarrollo personal como foco de estudio. En este enfoque es posible identificar los aportes de Rogers (1984), en su obra "El proceso de convertirse en persona", quien orienta el desarrollo del individuo a una subjetividad centrada en la multidimensionalidad de esferas que conforman la persona. "Mi técnica terapéutica", como ha denominado el autor a su trabajo, entrega herramientas sólidas para facilitar el desarrollo personal, canalizando de manera específica sugerencias y aportes al accionar educativo con niños que permitan la formación de habilidades sociales, de autoconocimiento y de valoración personal como plataformas de desarrollos posteriores, en los procesos evolutivos y frente a las experiencias vitales. Desde Latinoamérica, el equipo de investigadores en infancia y calidad de vida del Centro de Investigaciones en Desarrollo Humano de la Universidad del Norte (CIDHUM), en Barranquilla, Colombia, ha insistido en un trabajo situacional, experiencial y sustentado en los estudios fenomenológicos, presentando a la psicología comprensiva como un medio para "llegar a un conocimiento más concreto de la comprensión de la realidad de los niños que viven en sectores de pobreza a partir de lo que ellos mismos vivencian, es decir, mediante el estudio del niño desde el mismo niño" (Amar y Abello, 1998: 10). Su estudio manifiesta una incuestionable crítica a las corrientes psicológicas que han postulado el desarrollo infantil desde una perspectiva determinista y autómata, dado que insisten en que el ser biológico transciende hacia el ser social y cultural, definiendo el concepto de desarrollo humano (en relación a la psicología comprensiva) como "la realización del potencial biológico, social, y cultural del individuo mediante un proceso de autoconstrucción permanente con base en el establecimiento de una triple relación: consigo mismo, con los otros y con su ambiente" (Amar y Abello, 1998: 51). Algunas corrientes teóricas más actuales conjugan enfoques disciplinarios y alternativos que destacan el aporte de cosmovisiones de pensamiento orientales cuyos enfoques visualizan al individuo en dimensiones aún no incluidas del todo en el canon de la ciencia occidental. Entre ellas es posible identificar la psicología positiva y la psicología de la transpersonalidad.

El recorrido expuesto ha pretendido conglomerar algunos de los presupuestos, de un vasto cuerpo de investigaciones, que abordan el estudio del desarrollo humano, específicamente en la etapa infantil, desde diferentes ángulos teórico-metodológicos e influencias disciplinares. 


\subsection{PERSPECTIVAS TEÓRICAS SOBRE LA INTELIGENCIA}

Este subapartado centra su análisis descriptivo en el trabajo de Coll y Onrubia (2001), quienes han realizado un recorrido teórico que da cuenta de los cambios que se han producido en la forma de entender la inteligencia a partir de postulados y posiciones diversas, desde las teorías tradicionales hasta las desarrolladas en las últimas dos décadas.

En primer lugar, los autores reconocen al enfoque psicométrico de la inteligencia, o diferencial-psicométrico, y la perspectiva del procesamiento de la información dentro de las aproximaciones clásicas sobre inteligencia. La perspectiva diferencial psicométrica es la tradición de investigación que identifica a la inteligencia como un rasgo intelectual, o un conjunto de ellos, y permite establecer diferencias entre las personas. Esta tradición tiene sus orígenes, a finales del siglo XIX y principios del XX, con el trabajo de Albert Binet y Theodore Simon, quienes crearon la Escala Métrica de la Inteligencia, atendiendo al encargo de crear una prueba que identificara a los niños lentos o mentalmente retrasados que pudiesen beneficiarse de una enseñanza remedial, por el Ministerio francés de Instrucción Pública en 1904 (Shaffer, 2007). Este instrumento fue diseñado para medir destrezas que en ese momento se consideraban necesarias para el aprendizaje como: "atención, percepción, memoria, razonamiento numérico, comprensión verbal, entre otras" (Shaffer, 2007: 338).

La importancia del trabajo de Binet para el enfoque psicométrico se puede apreciar en tres dimensiones, según lo plantean Coll y Onrubia (2001). La primera dice relación con la posibilidad de medir rasgos psicológicos complejos que delimitan diferencias entre los individuos. La segunda dimensión señala la caracterización de la inteligencia como capacidades, habilidades o facultades que pueden estudiarse por separado, inicialmente, y establecer una conjunción al final del proceso para comprender su síntesis. En el caso de Binet y Simon, esta síntesis sería la "edad mental" (E.M.) que determina "la edad promedio con la que se corresponde la puntuación obtenida por el niño en la escala" (Coll y Onrubia, 2001; Boake, 2002); en este caso, un niño que fue evaluado en el test en una determinada edad promedio, su edad mental corresponde a esa edad promedio. Más tarde, en 1912, Stern propone la noción de "cociente intelectual" (C.I.) como medida de inteligencia o unidad estándar de comparación de rendimiento intelectual, y su fórmula corresponde a edad mental/edad cronológica x 100 (Boake, 2002; Coll y Onrubia, 2001; Shaffer, 2007). En último lugar, la propuesta de Binet y Simon manifiesta modificaciones y adaptaciones a lo largo del tiempo, pero aun así se mantiene parte de su esencia como base de los test estandarizados de inteligencia construidos posteriormente.

Para Charles Spearman (1927), el desempeño intelectual posee dos aspectos, un factor "g", general, correspondiente a una capacidad presente en todas las tareas intelectuales, y un factor "s" entendido como factor específico, capacidades específicas o especiales, propio de cada test individual que correlaciona con el factor "g". Por su parte Thurstone (1938), en su modelo multifactorial o multicomponente de la inteligencia, identificó siete capacidades mentales primarias que constituirían el concepto "g" de Spearman, correspondientes a: capacidad espacial, rapidez perceptual, razonamiento numérico, significado verbal, fluidez verbal, memoria y razonamiento inductivo (Shaffer, 2007).

Entre las propuestas más recientes de la perspectiva diferencial-semántico, es posible identificar "los modelos jerárquicos de la inteligencia" que representan un factor general (g) de capacidad intelectual, que se encuentra en la cúspide; otros factores principales y más especializados, que se organizan en un segundo nivel; y los factores específicos, que se 
encuentran en los niveles más bajos de la jerarquía. Según Coll y Onrubia (2001), Cattell considera la inteligencia fluida y la inteligencia cristalizada como dos factores que están por debajo del factor " $\mathrm{g}$ " en el orden jerárquico. La inteligencia fluida corresponde a la capacidad de las personas de pensar y razonar de manera abstracta, y se mide a través de tests de razonamiento inductivo basados en la resolución de problemas y analogías. La inteligencia cristalizada dice relación con el conocimiento, la carga cultural y las habilidades aprendidas, y se mide a través de tests de vocabulario, de información general o comprensión lectora. El modelo de Carroll (en Coll y Onrubia 2001) presenta tres estratos en donde el primero es el factor "g", el siguiente establece ocho habilidades específicas, y el tercero despliega un conjunto de habilidades vinculadas a los factores del segundo estrato.

Las influencias de esta perspectiva de medición de la inteligencia han sido notorias en el ámbito educativo. Entre ellas es necesario precisar la guía proporcionada por la edad mental para plantear programas de estudio destinados a estudiantes evaluados, según estos parámetros, como normales y retrasados (Shaffer, 2007). Por otro lado, la medida unitaria y absoluta de la capacidad intelectual, centrada en una concepción innatista de la génesis de las diferencias individuales y estática de la inteligencia, ha incidido considerablemente en la percepción que educadores manifiestan sobre el rendimiento escolar, como lo manifiestan los autores que orientan este apartado según su estudio:

Así, lo que en principio era una medida de inteligencia (el C.I.) se transforma en su esencia y los instrumentos de predicción del rendimiento escolar (los tets) se transforman en instrumentos de explicación de ese rendimiento: un alumno obtiene un determinado rendimiento escolar, bueno o malo, porque tiene un determinado CI, alto o bajo (Coll y Onrubia, 2001: 193).

Los argumentos evidenciados en los enunciados de la cita aludida parecen compartir las premisas explícitas en las conclusiones del estudio realizado por Bravo (1976), quién establece que tanto los resultados de las pruebas que miden la inteligencia, como la percepción evaluativa de los docentes respecto a esa medida, son la causa de la repetición de cursos en estudiantes de $4^{\circ}$ Básico. Así, estos factores condicionan el rendimiento escolar de los estudiantes.

Desde una óptica que intenta dar nuevas luces a la perspectiva diferencial psicométrica, las teorías del procesamiento de la información, tal como se mencionó al inicio del apartado, se enfocan en la identificación y comprensión de "los procesos cognitivos de selección, organización y procesamiento de la información implicados en el comportamiento inteligente, desarrollando modelos detallados de funcionamiento intelectual ante determinadas tareas" (Coll y Onrubia, 2001: 195). Las teorías del procesamiento de la información han entregado avances importantes a la investigación de las capacidades intelectuales en el contexto educativo, mediante la búsqueda de procedimientos y estrategias que permitan modificar y potenciar tales capacidades, articulando, de este modo, una concepción más dinámica de la inteligencia (Coll y Onrubia 2001).

En segundo lugar, desde una perspectiva más amplia y no unitaria, las inteligencias múltiples (Gardner 1995) y la inteligencia emocional (Goleman 1996) también forman parte de este nuevo cuerpo teórico y amplían el concepto tradicional de inteligencia (que se había desarrollado hasta la época), incorporando como objeto de estudio la multiplicidad de capacidades que el sujeto puede desarrollar en su acercamiento al conocimiento desde dimensiones diversas. Para Gardner, su autor, "la teoría de las inteligencias múltiples 
se organiza a la luz de los orígenes biológicos de la capacidad de resolver problemas", tratando, en su propuesta, las capacidades de carácter universal aludidas a la especie humana. Sin embargo, argumenta que "la tendencia biológica a participar de una forma concreta de resolver problemas tiene que asociarse también al entorno cultural" (1995:33). El autor genera una crítica a los modos tradicionales de concebir la inteligencia de manera genérica, y con un lente unidimensional para evaluar la mente de las personas, los cuales han sido transferidos al sistema educativo institucionalizado, estableciendo lo que Gardner ha denominado una "visión uniforme" dentro de la organización del curriculum escolar, situado éste, mayoritariamente, en ciertas competencias de orden lógico, lingüístico y matemático. Por el contrario, su marco analítico perfila "una visión pluralista de la mente humana que reconoce muchas facetas distintas de la cognición” (Gardner 1995:24), desarrollando un trabajo que involucra diversas capacidades, las cuales ha conglomerado en siete tipos de inteligencias. Goleman, desde su filtro disciplinar, también replantea el paradigma del pensamiento lógico, racional y adaptativo representado en el coeficiente intelectual, apostando por un reposicionamiento del desarrollo afectivo emocional que resulta ser el andamiaje que sostiene el desarrollo del sujeto en las experiencias vitales, siendo capaz de diseñar su proceso de autoconocimiento a través de la comprensión y el control emocional. Esta capacidad de manejo emocional, que hasta la hora se consideraba subyugada a la razón, permite al individuo hacer conscientes los estados y niveles complejos del desarrollo de sentimientos y emociones para establecer actitudes, frente a las circunstancias de vida, que otorgan organización a la conducta, desde el reconocimiento personal hasta el reconocimiento de un otro legítimo, en la articulación de los procesos vinculares.

En último lugar, la teoría triárquica de la inteligencia planteada por Stenberg (1985), basada en las teorías del procesamiento de la información, también reconceptualiza la noción tradicional de las capacidades intelectuales, considerando a la inteligencia en relación con entornos reales del mundo de la vida y cercanos a la cotidianidad. Es el sujeto que participa de esos entornos el que despliega capacidades intelectuales para adaptar, modificar o transformar, de manera planificada y organizada, aspectos relativos a la interacción que construye en, y con, esos espacios. Esta teoría pone de relieve tres aspectos -componentesdel comportamiento inteligente. El primer componente -el contextual- establece que la evaluación de una conducta inteligente debe ser observada en un contexto. De este modo, existe una relación bidireccional entre las capacidades intelectuales del individuo y el contexto en donde éstas se desarrollan, afectándose mútuamente. El segundo componente -el experiencial- se ocupa de la relación inteligencia-experiencia. En este sentido, la inteligencia se manifiesta al afrontar nuevas tareas o desafíos en situaciones concretas, o en la automatización de la conducta inteligente frente a tareas conocidas que requieran la puesta en escena de capacidades ya desarrolladas. El tercer componente -integral, componencial o de procesamiento de la información- hace referencia a los procesos cognitivos que tienen lugar en la resolución de problemas a partir de la formulación, ejecución y monitoreo de estrategias para resolverlos, organizando la actividades cognitivas que posibilitan este proceso (Coll y Onrubia, 2001; Shaffer, 2007).

Los trabajos más recientes asociados a esta teoría han incorporado tres nuevos componentes a la caracterización de la inteligencia. Esta noción se codifica a través del significado del éxito experimentado personalmente en las transiciones vitales, y se reconoce como "inteligencia exitosa". El aspecto analítico - primer componente- se utiliza para 
resolver problemas; el segundo -aspecto creativo-, para decidir qué problemas resolver; y el tercero -de tipo práctico-, para hacer operativas las soluciones (Coll y Onrubia, 2001).

En último lugar, aportaciones más recientes han construido una nueva perspectiva de la actividad cognitiva (inteligencia), considerando como punto de partida su naturaleza distribuida. Esta concepción sostiene que la actividad del pensamiento no se canaliza en los procesos mentales de un individuo al margen del contexto, sino que hace referencia a la actividad instanciada de una persona en un contexto situacional que le otorga sentido y cuya naturaleza es social. Sus aportaciones al ámbito pedagógico han permitido construir nuevas propuestas de acción educativa, como el desarrollo de comunidades de aprendizaje, el aprendizaje colaborativo, la estructura dialógica de construcción de conocimiento compartido, entre otras.

\section{UN MODELO TRANSVERSAL DE APRENDIZAJE BASADO EN EL CAMBIO GENERATIVO DE DBM®}

De acuerdo con el análisis expuesto, este trabajo propone un modelo transversal que visibiliza la relación compleja y dinámica entre aprendizaje, inteligencia y desarrollo, basado en el DBM®. El DBM®, como su autor lo define, "es una integración práctica de una metodología formal de modelado y está diseñada para funcionar con el accionar cotidiano". Formalmente, es una integración de tecnología conductual, mucho de ella basada en las habilidades nucleares de la PNL (Programación Neurolingüística), que organiza los procedimientos a seguir. De manera conjunta, se conceptualiza como una metodología holística de modelado, dado que focaliza su atención en los procesos que conciben la conducta, y una epistemología cuyos principios están basados en el trabajo de Gregory Bateson (McWhirter 1999, 2000).

De acuerdo con su autor, el DBM® es una herramienta metodológica que facilita el cambio, de este modo la razón principal de su aplicación es "trabajar con las personas y, mediante la exploración y educación en conjunto, mejorar nuestras habilidades y la aplicación de ellas en el crear y seleccionar las acciones más relevantes para nuestro crecimiento y desarrollo como seres humanos" (McWhirter 1999:3). Como herramienta de análisis, utiliza una planilla vital que identifica áreas de la experiencia personal y niveles de procesamiento de esta experiencia, para modelar la complejidad humana. El modelado conductual se basa en los principios del aprendizaje social desarrollado por Vygotski (1979), específicamente en la Zona del Desarrollo Proximal, en donde cobra importancia el aprendizaje mediado por el entorno, a través de relaciones intersubjetivas, y de la práctica del lenguaje como eje clave en la configuración de procesos sociales y culturales que darán forma y sentido al desarrollo. En este caso, el DBM ${ }^{\circledR}$ se construye como una herramienta metodológica que permite facilitar el cambio, como proceso de aprendizaje mediado en el contexto del desarrollo personal, y para ello reconoce cuatro áreas de facilitación. La primera dice relación con facilitar el uso, y corresponde al fomento de las habilidades dentro del contexto de lo que la persona es capaz de hacer en el momento de aprendizaje actual. La segunda -facilitar el aprendizaje-, hace alusión a aquello que está en vías de aprenderse dentro de la zona de desarrollo de la persona. La tercera, codificada como facilitar el aprender a aprender, se establece en la zona del desarrollo de la persona y da cuenta de los procesos que focalizan el aprendizaje como tal. La cuarta, y última dimensión, correspondiente a 
facilitar el desarrollo, se establece más allá de la zona de desarrollo de la persona, es decir más allá del aprendizaje actual, e inspira la aparición de nuevas posibilidades.

Uno de los principios que sustenta esta metodología de cambio es el de la fractalidad que, como su autor lo menciona, otorga la posibilidad de describir detalles en potencia al infinito en un espacio finito. De este modo, los indicadores fractales pueden resaltar la flexibilidad, relevancia y utilidad de los modelos implicados en el DBM® (McWhirter 1999). Estos modelos, desarrollados por la metodología en cuestión, han incorporado propiedades básicas de la fractalidad para organizar de manera compleja sus estructuras de análisis, en las que se encuentran la autosimilitud de la estructura (en cada aspecto que considere la estructura), la complejidad infinita en espacio finito, y las causas simples que conducen a comportamientos complejos. El análisis en niveles permite identificar la organización de la estructura con mayor especificidad, dado que el detalle amplifica las distinciones que conforman lo analizado y articula, como ya se ha mencionado, niveles de mayor delicadeza y complejidad.

Como metodología, incorpora tres aspectos que otorgan orientación a la exploración de las experiencias vitales. El primero de ellos corresponde a la Epistemología, que se basa en los principios que rigen la experiencia personal y otorgan la base cognitiva que fundamenta el accionar individual; ésta se consigna en el nivel del POR QUÉ. En segundo lugar, la Metodología delimita los procesos intervinientes en la experiencia vital y en el desarrollo de la conducta, y se identifica en el nivel del CÓMO. En último lugar, la Tecnología, que da cuenta del repertorio conductual que se sitúa en cada aspecto de la experiencia vital, y se codifica en el nivel de QUÉ. La relación interniveles en la gestión de procesos de cambio, para su visibilización y ocurrencia durante el aprendizaje, puede ser organizada siguiendo los principios fractales a los que hace referencia McWhirter $(1999,2000,2011)$, a través de una planilla vital, según lo muestra la siguiente figura (como se mencionó anteriormente se identifican áreas de experiencia personal y el nivel de procesamiento de la experiencia):

Figura 1: Planilla vital para identificar áreas de la experiencia personal y niveles de procesamiento de esta experiencia ${ }^{6}$

\begin{tabular}{|l|l|l|}
\hline Nivel DBM $®$ & Nivel Fractal & \multicolumn{1}{c|}{ Breve Descripción } \\
\hline Epistemología & Por qué & Por qué funcionan los principios \\
\hline Principios & Cómo & Cómo están secuenciados los principios \\
\hline Por qué & Qué & Qué principios están involucrados \\
\hline Metodología & Por qué & Por qué los procesos funcionan en el orden en el que funcionan \\
\hline Procesos & Cómo & Cómo están secuenciados los procesos \\
\hline Cómo & Qué & Qué procesos están operando \\
\hline Tecnología & Por qué & Por qué en un orden determinado \\
\hline Procedimientos & Cómo & Cómo hacerlo \\
\hline Qué & Qué & Qué hace que funciona \\
\hline
\end{tabular}

Basado en McWhirter, 1999. 
De acuerdo con las propuestas del modelado, entre sus principales objetivos se advierte la identificación de necesidades precisas, de una situación, para crear respuestas mediante el aprendizaje, y a través del proceso de cambio. Para McWhirter, la comprensión del mundo se construye a través del aprendizaje situado en las experiencias vitales, según lo expresa en la siguiente afirmación "Nuestra comprensión del mundo dictará cómo respondemos a los retos de la vida y, también, qué tipo de aprendizaje creemos no sólo apropiado, sino también posible" (McWhirter 2000:2). De acuerdo con el autor, un aspecto fundamental de la aplicación de la metodología refiere a identificar el por qué se espera producir cambios. De este modo, el sujeto puede examinar posibilidades de soluciones a necesidades particulares, o plantear procesos de cambio personal, una vez que considere la selección dentro de una gama de opciones que organizan sus modelos de comprensión del mundo.

En el sentido expuesto, es necesario considerar un equilibrio entre la relevancia que tienen las elecciones y el aspecto práctico que implica el elegir (acción). El aumento de elecciones (ante una situación en que se añaden más y más elecciones) podría imposibilitar la capacidad de decidirse por una de ellas, si es que no se clarifican elementos intervinientes en el proceso. Para precisar este aspecto, el autor distingue tres niveles de elección para evaluar las decisiones que proponen procesos de cambio. La primera corresponde a la elección, que guarda relación con la creación y selección del tipo, dentro de una gama de opciones en algún aspecto determinado a evaluar (es más genérica). El segundo nivel hace referencia a la opción, que establece la creación y selección del ejemplo dentro del tipo. El tercer nivel delimita las alternativas, que establecen la creación y selección del comportamiento específico dentro del ejemplo. A modo de ejemplo sencillo, se puede mencionar las alternativas dentro de una carta de un restaurante (tipo de elección, que son variaciones dentro del mismo elemento o cosa). En tanto que la opción, tipo de elección de la gama de alternativas, puede ejemplificarse en la elección de un tipo de restaurante, y menú, dentro de un elenco de opciones. En tercer lugar la elección, en cuanto a gama de opciones, establece elecciones más amplias, como por ejemplo elegir ir a cenar dentro de una gama de elecciones posibles como ir al cine, a un baile, etc.

Asumir el control del proceso de aprendizaje a nivel personal, según esta propuesta, se facilita en tanto estén disponibles los tres niveles de elección frente a la toma de decisiones dentro del contexto de la experiencia vital. Desde esta perspectiva, es necesario resaltar las distinciones procesuales que se postulan en la gestión del cambio a partir de los niveles mencionados. De acuerdo con el DBM® (McWrirter 1999,2011), los beneficios desarrollativos que emergen en la elección de una gama de posibilidades, son distintos en cada una de las dimensiones expuestas. Es por tanto que, dentro de las decisiones conductuales que dan sentido a los procesos de cambio, el contar con más alternativas aumenta la Variabilidad en potencia, de igual modo que el tener un mayor número de opciones incrementa la Flexibilidad en potencia. Finalmente, el disponer de un mayor número de elecciones aumenta la posibilidad de Adaptabilidad frente a las circunstancias y tránsitos vitales para facilitar procesos de cambio.

La propuesta a la que alude este trabajo integra los aspectos señalados -los que forman parte del Modelo DBM®-, específicamente para sistematizar, a través del proceso de aprendizaje, niveles de procesamiento de esta experiencia. Esta sistematización pretende organizar la relación bidireccional entre aprendizaje e inteligencia en función del desarrollo del sujeto y, de este modo, incluye aspectos centrales de los postulados 
manifiestos por la teoría triárquica de la inteligencia, que a decir de Coll y Onrubia precisa "considerar la inteligencia en relación con entornos del mundo real, relevantes para la vida cotidiana, y en términos de su aplicación a la adaptación, modificación y selección de dichos entornos de forma organizada y planificada por parte de la persona (2001: 198). Por tanto, esta propuesta considera el componente contextual, componencial (procesamiento de la información) y experiencial de esta teoría ${ }^{7}$.

La comprensión del mundo, como ya se ha mencionado, se construye a través de los aprendizajes que se sitúan en las experiencias de la vida. La evidencia de las orientaciones que permiten identificar cuáles son los aprendizajes posibles o viables para dar respuesta al proceso de comprensión o representación del mundo, da como resultado un proceso de cambio. En tanto el sujeto se hace consciente del procesamiento instanciado en el modo en que procede el cambio, y lo orienta hacia el desarrollo de su potencial personal, el aprendizaje se sitúa en el cambio generativo (cambio que construye el sujeto).

Como primer aspecto, el modelo transversal de aprendizaje basado en el cambio generativo integra los tres niveles de exploración de las experiencias vitales, los cuales han sido descritos en dimensiones que permiten llegar a una aproximación de los alcances del proceso de aprendizaje en los niños (estudiantes) que participan de él. El nivel del QUÉ, corresponde a la dimensión de tecnología conductual y busca establecer el patrón de la conducta inteligente del sujeto. Este se considera en el Nivel del Hacer, manifiesto a través del Logro/Efecto del aprendizaje dentro del contexto disciplinar en donde se sitúa. El nivel del CÓMO organiza la dimensión metodológica, e identifica la forma en que el sujeto gestiona su conducta a través del reconocimiento de las habilidades implicadas en el proceso de aprendizaje (cognitivas, sociales, físicas, etc.). Además, este nivel hace referencia al procesamiento afectivo que se manifiesta en el Nivel del Sentir y corresponde a la Afiliación/Afecto. En último lugar, el nivel del POR QUÉ organiza la dimensión epistemológica y permite visualizar el posicionamiento del sujeto frente a las circunstancias, denotando el impacto sobre las acciones situadas en su experiencia vital. Además, construye significados a nivel del status socio-familiar, las acciones personales y el sentido de la vida. Para efectos de este trabajo, se codifica, según lo manifiesta el modelo de DBM®, como el Nivel del Significar que se relaciona con el Posicionamiento/Impacto. De igual modo, se ha incorporado un nivel que correlaciona con el Posicionamiento/Impacto, dado que establece directa relación con la dimensión Epistemológica en el proceso de aprender. En este caso, éste se ha representado a través del nivel del PARA QUÉ, dado que articula, en el sujeto que aprende, componentes metacognitivos que explicitan un monitoreo y una toma de conciencia de los procesos situados en el aprendizaje. Además, este modelo considera el principio de fractalidad, distinguiendo que cada nivel se organiza de acuerdo con su autosimilitud en la estructura y con niveles de mayor delicadeza y complejidad. 
Figura 2: Representación fractal de la experiencia de aprendizaje según niveles de procesamiento

\begin{tabular}{|c|c|c|c|}
\hline Dimensión & Nivel & $\begin{array}{l}\text { Procesamiento } \\
\text { de la experiencia }\end{array}$ & Nivel Fractal \\
\hline \multirow{3}{*}{ QUÉ } & \multirow{3}{*}{$\begin{array}{l}\text { Hacer } \\
\text { Logro/Efecto }\end{array}$} & \multirow{3}{*}{$\begin{array}{l}\text { ¿Qué hago para aprender? } \\
\text { ¿Qué aprendo? }\end{array}$} & $\begin{array}{l}\text { Qué: ¿qué hace que funcione esta acción } \\
\text { de aprender? }\end{array}$ \\
\hline & & & $\begin{array}{l}\text { Cómo: ¿Cómo realizo/hago (que fun- } \\
\text { cione) esta acción de aprender? }\end{array}$ \\
\hline & & & $\begin{array}{l}\text { Por qué: ¿Por qué la acción de aprender } \\
\text { funciona en un orden determinado? }\end{array}$ \\
\hline \multirow{3}{*}{ COMO } & \multirow{3}{*}{$\begin{array}{l}\text { Procesamiento } \\
\text { Afiliación/Afecto }\end{array}$} & \multirow{3}{*}{$\begin{array}{l}\text { ¿Cómo aprendo? } \\
\text { ¿Cómo me siento aprendiendo? }\end{array}$} & $\begin{array}{l}\text { Qué: ¿Qué procesos están operando en } \\
\text { el aprendizaje? }\end{array}$ \\
\hline & & & $\begin{array}{l}\text { Cómo: ¿Cómo están secuenciados esos } \\
\text { procesos? }\end{array}$ \\
\hline & & & $\begin{array}{l}\text { Por qué: ¿Por qué los procesos funcio- } \\
\text { nan en el orden que funcionan? }\end{array}$ \\
\hline \multirow{3}{*}{$\begin{array}{l}\text { POR QUÉ } \\
\text { PARA QUÉ }\end{array}$} & \multirow{3}{*}{$\begin{array}{l}\text { Significar } \\
\text { Posicionamiento/ } \\
\text { Impacto }\end{array}$} & \multirow{3}{*}{$\begin{array}{l}\text { ¿Por qué aprendo de ese modo? } \\
\text { ¿Para qué aprendo? }\end{array}$} & $\begin{array}{l}\text { Qué: ¿Qué significados/sentidos están } \\
\text { involucrados en mi aprendizaje? }\end{array}$ \\
\hline & & & $\begin{array}{l}\text { Cómo: ¿Cómo están secuenciados esos } \\
\text { significados/sentidos en mi aprendizaje? }\end{array}$ \\
\hline & & & $\begin{array}{l}\text { Por qué: ¿Por qué esos significados/sen- } \\
\text { tidos están involucrados (funcionan) en } \\
\text { mi aprendizaje? }\end{array}$ \\
\hline
\end{tabular}

La primera dimensión, el QUÉ, se hace visible en el Nivel del Hacer y da cuenta del Logro/Efecto, en tanto el sujeto reconoce la efectividad de su accionar en el aprendizaje y el logro que este sitúa en su desarrollo, potenciando aspectos motivacionales intrínsecos. Se correlaciona con las distinciones que organizan los nodos de información con los que se relaciona el acto de aprender, atendiendo a principios y contenidos de las disciplinas en donde se explicitan tales distinciones. A nivel fractal, es posible organizar la estructura de esta dimensión identificando los aspectos que hacen que el acto de aprender oriente la experiencia del estudiante. Además, se incluye el modo en que operan los aspectos mencionados (en el acto de aprender) y el orden en que estos se establecen.

La segunda dimensión -el CÓMO-, identifica los procesos que están operando en el acto de aprender. En esta dimensión, es posible distinguir las habilidades del pensamiento relativas a los niveles de transferencia y codificación (teorías del procesamiento de la información), a través de procesos sistemáticos y complejos como la observación y descripción, construcción de explicaciones e interpretaciones, razonamiento con evidencia, establecimiento de conexiones, consideración de puntos de vista y perspectivas, elaboración de conclusiones, etc. (Ritchhart et al, 2014). También incluye habilidades expresivas orales, corporales, artísticas, entre otras. Es necesario mencionar que, conjuntamente con las habilidades descritas, el desarrollo afectivo es fundamental en esta dimensión, en tanto que el estudiante otorga significado emocional a la experiencia vinculada al acto de aprender, lo que constituye un posicionamiento de valía personal a nivel afectivo y conceptual. Este aspecto está relacionado con la siguiente dimensión debido al Impacto (significados) que 
genera la acción del sujeto de modo intersubjetivo. A nivel fractal, organiza los procesos que operan en el aprendizaje, la secuencia de esta operacionalización y la justificación de su funcionamiento.

La tercera y última dimensión -el POR QUÉ y PARA QUÉ, representada en el Nivel del Significar, hace referencia a los modelos cognitivos que organizan el Impacto y el Posicionamiento del sujeto frente a la experiencia de aprendizaje. En esta dimensión, es posible evidenciar los procesos de interiorización y apropiación ${ }^{8}$, en donde la interacción otorga sentido a la transición de los elementos culturales a las representaciones conceptuales elaboradas por los sujetos, cuyos patrones de relación sistematizan la negociación de significados adscritos a los procesos sociales en donde se instancia esa interacción. Los contenidos culturales de los que participa el estudiante pueden ser identificados a través de los esquemas representacionales con que comunica la comprensión del sentido de realidad, de acuerdo con las nomenclaturas clasificatorias configuradas a través de los filtros perceptivos que significan esos esquemas. En este sentido, la representación de la experiencia vital se codifica en el lenguaje. Según este parámetro, es relevante en este nivel recoger el conocimiento previo del estudiante a partir de sus modelos de comprensión de la realidad, y desde ahí focalizar los procesos que están operando en el acto de aprender en un contexto específico.

De manera conjunta, el PARA QUÉ monitorea los tres niveles en que se procesa la experiencia, para fortalecer la toma de conciencia en las decisiones que postularán a cambios en el desarrollo mediante el aprendizaje.

De acuerdo con la teoría triárquica de la inteligencia desarrollada por Sternberg, según lo manifiestan Coll y Onrubia (2001), esta (la inteligencia) puede ser entendida a través de la relación "mundo externo /interno", mediatizada por la experiencia en el desarrollo del individuo. Siguiendo esta premisa, los niveles de procesamiento de la experiencia de aprendizaje, propuestos en el modelo expuesto, permiten explorar la diversidad de capacidades implicadas en el comportamiento inteligente. Es así que el nivel del Hacer y del Significar (Qué, Por qué, Para qué) codifican el componente contextual de la conducta inteligente, dado que el aprendiz visibiliza la interacción manifiesta con el mundo. Además, esta interacción evidencia los procesos de adaptabilidad y de transformación generados en la relación sujeto-entorno sociocultural, a través del cambio.

En segundo lugar, el nivel de Procesamiento (Cómo) organiza el componente componencial relativo a los procesos de adquisición y ejecución en la resolución de problemas, destacando las habilidades cognitivas que se visibilizan durante la experiencia del aprendizaje, las que son organizadas de manera secuencial y según su funcionamiento. De manera particular, el modelo transversal de aprendizaje enfatiza las habilidades cognitivas, corporales, artísticas, de desarrollo afectivo emocional, entre otras (de acuerdo a la percepción que el estudiante manifiesta en relación a la experiencia de aprendizaje).

En último lugar, el componente experiencial puede expresarse en los tres niveles del modelo dado que el estudiante reconoce cómo la experiencia afecta la inteligencia, y viceversa. Sin embargo, es el nivel del Hacer (Qué) es el que permite explorar el logro que valida la efectividad de la acción en la experiencia de aprendizaje, cualificando, de este modo, la conducta inteligente y los procesos de cambio que sostiene.

\footnotetext{
Herramientas que han servido a la teoría sociocultural del aprendizaje para identificar el origen social del funcionamiento mental en el individuo (Cubero y Luque, 2001).
} 
Como segundo aspecto, el modelo transversal de aprendizaje basado en el cambio generativo reconoce las posibilidades de elección que significan, orientan y sostienen el cambio. Una vez que el aprendiz establece el mapa de referencia mediante el proceso que explicita su aprendizaje (según el esquema de representación del procesamiento de la experiencia de aprendizaje), puede organizar sus decisiones en tanto que conjuga las alternativas, las opciones y las elecciones manifiestas en el acto de aprender. De acuerdo con la propuesta de McWhirter, el tipo de elección facilitará las decisiones que promueven el cambio. Es así que si el estudiante reconoce las alternativas en el proceso de aprendizaje, tendrá sentido la variabilidad en que se sitúa el proceso. Del mismo modo, si reconoce las opciones que organizan esas alternativas podrá acceder a la flexibilidad en el acto de aprender; y en último lugar, si accede a las elecciones que asimilan las opciones y alternativas, organizadas a nivel sistémico, facilitará los sistemas adaptativos que posicionan los procesos de cambio para su potencial desarrollo personal, social y cultural. ${ }^{9}$

Como último aspecto, es necesario destacar el componente social en el desarrollo del modelo propuesto, dado que el procesamiento de la experiencia puede organizarse en espacios de aprendizaje colaborativo, de auto y co-evaluación, donde las posibilidades de desarrollo conjugan procesos de cambio a nivel personal y de manera colectiva, según el contexto en donde se implemente.

\section{CONCLUSIONES}

Los resultados de la discusión expuesta en este trabajo permiten caracterizar a la Inteligencia más que como un predictor del rendimiento escolar, según su condición de medida psicométrica y unitaria, como un componente de la relación compleja y dinámica entre aprendizaje y la potenciación del desarrollo del sujeto. En este sentido, la conducta inteligente evidencia habilidades y capacidades que operan en planos de acción concreta y en contextos diversos, en donde la experiencia asume un rol protagónico.

El vasto cuerpo de investigaciones que han aportado al estudio del desarrollo infantil y a la conceptualización de la inteligencia, a partir de enfoques cognitivos, cognitivo socialesevolutivas y los modelos ecológico-sistémicos-comprensivos, encuentran un eje común en la relación intersubjetiva que construye el individuo entre los procesos psicológicos de orden superior (mundo interno), los contextos socioculturales (mundo externo) y la experiencia que mediatiza sus correlaciones. Los recursos de interiorización y de apropiación explican la afectación mutua de estos sistemas en donde los modelos mentales, configurados por el individuo, son construidos a partir de los códigos aprendidos en la práctica social. Esta práctica significa la experiencia que recoge los elementos de la cultura, como mecanismo de apropiación e interiorización. De igual modo, los contextos de interacción pueden ser modificados por el sujeto de acuerdo a sus intereses, y a las formas que otorgan sentido a la comprensión de esos espacios de interacción. Particularmente, los enfoques teóricos que recientemente han estudiado la inteligencia, proponen explicaciones no unitarias de la misma, y muestran una relación bidireccional entre aprendizaje y desarrollo, a diferencia de la perspectiva diferencial psicométrica evidenciada en las aproximaciones clásicas.

Este aspecto será trabajado con mayor detalle en un posterior artículo. 
Las influencias de estas corrientes teóricas han demarcado orientaciones específicas en la implementación de programas educativos para la formación académica de los estudiantes. En el caso del trabajo de Bravo (1976), correspondiente al artículo del cual este trabajo hace referencia, la medida psicométrica de la inteligencia ${ }^{10}$, se consideraba un factor predictor del rendimiento escolar y de la repitencia de curso en niños de cuarto año básico, de acuerdo con los resultados obtenidos en el grupo en estudio. Además, el autor manifiesta como hipótesis, en su proyección investigativa, que la evaluación subjetiva de los docentes acerca del rendimiento escolar, según el parámetro unitario de la inteligencia, incide en la aprobación o reprobación de un curso. El cuestionamiento de este aspecto pone en tela de juicio la operatividad del sistema escolar en ese minuto.

Este trabajo no coincide con el sistema de medición que define el rendimiento escolar del estudiante basado en la psicometría de la inteligencia. Sin embargo, destaca la postura que posiciona a los investigadores desde un ángulo que advierte atisbos de una nueva concepción de aprendizaje basada en el desarrollo de habilidades del pensamiento y en una evaluación centrada en el rendimiento del estudiante y no en la percepción del docente.

Finalmente, la propuesta analítica de este estudio ha invitado a reconceptualizar la noción de repitencia, que centra su orientación semántica en el déficit de los resultados del rendimiento académico, por una noción compleja y dinámica que se significa en la relación: aprendizaje, desarrollo e inteligencia. Esta concepción de aprendizaje focaliza el cambio generativo como potenciador del desarrollo en espacios diversos de interacción, cuya orientación evaluativa emerge en el diagnóstico y autoconciencia de los procesos intervinientes en el acto de aprender.

\section{REFERENCIAS BIBLIOGRÁFICAS}

Amar, J. y Abello R. (1998). El niño y su comprensión del sentido de la realidad. Barranquilla: Uninorte.

Atkinson, R. y Shiffrin, R. (1968). Human memory: A proposed system and its control processes. En K. Spence y J. Spence (Ed.), The psychology of learning and motivation: Advances in research and theory (pp. 90-195). Orlando FL: Academic Press.

Ausubel, D. P. (1963). The psychology of meaningful verbal learning: An introduction to school learning. Nueva York: Holt, Rinehart y Winston.

Bandura, A. (1986). Social foundations of thought and action. A social cognitive theory. Englewood Cliffs, NJ: Prentice- Hall.

Bandura, A. (1989). Social cognitive theory. En R. Vista (Ed.), Annals of child development (vol 6, pp. 1-60). Greenwich,CT: JAI Press.

Bandura, A. (1991). Social cognitive theory of moral thought and action. En W. Kurtines y J. Gewirtz (Ed.), Handbook of moral behavior and development (vol 1, pp. 45- 103). Hillsdale. NJ: Erlbaum. Beard, R. (1971). Psicología evolutiva de Piaget. Buenos Aires: Kapelusz.

Berger, P. y Luckmann, T. (1986). La construcción social de la realidad. Buenos Aires: Amorrortu.

Boake, C. (2002). From the Binet-Simon to the Weschsler-Bellevue: Tracing the history of Intelligence Testing. Journal of Clinical y Experimental Neuropsychology, vol. 24, 383-405.

Bowlby, J. (1993). El vínculo afectivo. Barcelona: Paidós. 1969.

. (1985). El papel de la experiencia de la infancia en el trastorno cognitivo. En M. Mahoney y

10 En el contexto del sistema educativo en Chile entre los años 1972 y 1976. 
W. Freeman (Eds.), Cognición y Psicoterapia (pp. 203- 224). Buenos Aires: Paidós. Paidós.

(1988). Una base segura. Aplicaciones clínicas de una teoría del apego. Buenos Aires:

Bravo, L. (1976). La repetición de cursos en Educación Básica: Estudio Evaluativo del sistema de alumnos con distinto número de repitencias. Estudios Pedagógicos, 1, 7-17.

Bronfenbrenner, U. (2005). Making human beings human. Thousand, CA: Sage . (1995). The bioecological model from a life course perspective: Reflections of a participant observer. En P. Moen, G. H. Elder, Jr. Y K. Luscher (Eds.), Examining lives in context (pp. 599618). Washington, DC.: American Pychological Association. (1993). The ecology of cognitive developmental: Research models and fugitive findings. En R. H. Wozniak y K. W. Fisher (Eds.), Developmental in context (pp. 3-44). Hillsdale. NJ: Erlbaum. . (1979). The ecology of buman development: Experiments by nature and design. Cambridge. MA: Harvard University Press.

Bruner, J. (1985). Models of the learner. Educational Researcher, vol. 14 (6), 5-8. . (1983). Child's talk: Learning to use language. Nueva York: Norton. . (1964). The course of cognitive growth. American Psycbologist, 19, 1-15.

Cantón, J., Cortés, M. y Catón, D. (2011). Desarrollo socioafectivo y de la personalidad. Madrid: Alianza.

Craik, F. I. M. y Tulving, E. (1975). Depth of processing and the retention of words in episodic memory. Journal of experimental Psycbology: General, 104, 268-294

Coll, C. y Martí, E. (2001). Aprendizaje y desarrollo: la concepción genético-cognitiva del aprendizaje. En C. Coll, A. Marchesi y J. Palacios, Desarrollo Psicológico y Educación (pp. 67-88). Madrid: Alianza.

Coll, C. y Onrubia, J. (2001). Inteligencia, inteligencias y capacidad de aprendizaje. En C. Coll, A. Marchesi y J. Palacios, Desarrollo Psicológico y Educación (pp. 189-210). Madrid: Alianza.

Cubero, R. y Luque, A. (2001). Desarrollo, educación y educación escolar: la teoría sociocultural del desarrollo y del aprendizaje. En C. Coll, A. Marchesi y J, Palacios, Desarrollo Psicológico y Educación (pp. 137-156). Madrid: Alianza.

Coll, C., Marchesi, A. y Pallacios, J. (2001). Desarrollo Psicológico y Educación. Madrid: Alianza.

Douglas, M. (1973). Pureza y Peligro. Un análisis de los conceptos de contaminación y tabú. Madrid: Siglo Veintiuno.

Ebbinghaus, H. (1913). Memory. A contribution to experimental psycology. New York City: Teachers College Columbia University.

Erikson, E. (2000). El ciclo vital completado. Barcelona: Paidós . (1993). Infancia y Sociedad. Buenos Aires: Hormé, SA.

Freud, S. (1960). A general introduction to phychoanalysis. Nueva York: Washington Square Press (Obra original publicada en 1935).

. (1930). Tree contributions to the theory of sex. Nueva York: Nervous and Mental Disease Publishing Co (obra original publicada en 1905). . (1933). New introductory lectures in psychoanalysis. Nueva York: Norton.

Gadner, H. (1995). Inteligencias Múltiples. Barcelona: Paidós

Gagné, R. M. (1985). The conditions of learning (4a. ed). Nueva York: Holt, Rinehart y Winston.

Gagné, R. M. y Briggs, L. J. (1979). Principles of instructional design (2a . ed). Nueva York: Holt, Rinehart y Winston.

Gesell, A. (1933). Maturation and the patterning of behavior. En C. Murchison (Ed), A handbook of child psychology (pp. 209-235). Worcester, MA: Clark University Press

Guiñez, M y Martínez, E. (2015). Mediación lectora y primera infancia: Construcción de sentidos subjetivos e identitarios. Estudio de caso en niños de $3^{\circ}$ y $4^{\circ}$ de educación básica del Colegio Adventista de Valdivia. Estudios Pedagógicos, 41, n Especial, 115-134. 
Estudios Pedagógicos, Número Especial 40 años: 11-30, 2016

RECONSTRUCCIÓN CONCEPTUAL DEL SISTEMA DE REPITENCIA EN EDUCACIÓN BÁSICA: UNA PROPUESTA DE APRENDIZAJE MEDIADO BASADA EN EL CAMBIO GENERATIVO

Goleman, D. (1996). La inteligencia emocional. Buenos Aires: Javier Vergara.

Klein, M. (1932). The Psycho-Analysis of Children. London: Hogarth.

Kohlberg, L. (1992). Psicología del desarrollo moral. Bilbao: Desclee de Brower.

Mc Whirter, J. (2011). Behavioral Remodelling. Advancing NLP's Linguistic Model. En Hall, M. y Charvet, S. Innovations in NLP (pp. 94-113). UK: Crownhouse Publications

Mc Whirter. J. (2000). Facilitar el Cambio. Diploma en Hipnosis Eriksoniana. Master de Intervención Psicológica con DBM®: desde el Desarrollo Personal al Trabajo con el Cliente. Manual del Máster.Valencia, España

Mc Whirter, J. (1999). Re-Modelling. N.L.P Part One. Models and Modelling. Revista ANLP Rapport.

Morris, C.D. Bransford, J.D. y Franks, J. J (1977). Levels of processing versus transfer-appropriate processing. Journal of verbal learning and verbal Behavior, 16, 519-533.

Moscovitch, M. y Craik, F. I .M. (1976). Depth of processing, retrieval cues, and uniqueness of encoding as factors in recall. Journal of verbal learning and verbal Behavior, 15, 447-458.

Nelson, T. O. (1977). Repetition and depth of processing. Journal of verbal learning and verbal Behavior, 16, 151-171.

Palladino, E. (2009). Infancia, sociedad y educación. Desarrollo de la subjetividad. Buenos Aires: Espacio Editorial.

Papalia, D. y Wendkos Olds, S. (1988). Desarrollo Humano. México: Mc Graw-Hill.

Piaget, J. (1973). Seis estudios de psicología. Barcelona: Seix Barral. . (1965). The moral judgment of the child. Nueva York: Free Press.

(1961). La formación del símbolo en el niño. Imitación, Juego, sueño, imagen y representación. México: Fondo de cultura económica.

. (1954). The construction of reality in the child. Nueva York: Basic Books.

Postman, L. (1961). The present status of interference theory. En C. N. Cofer (Ed.), Verbal learning and verbal behavior (pp. 152-179). Nueva York: McGraw-Hill.

Ritchhart, R., Church, M. y Morrison, K. (2014). Hacer visible el pensamiento. Cómo promover

el compromiso, la comprensión y la autonomía de los estudiantes. Buenos Aires: Paidós.

Rogers, C. (1984). El proceso de convertirse en persona: Mi técnica terapéutica. Buenos Aires: Paidós.

Shaffer, D. y Kipp, K. (2007). Psicología del Desarrollo: de la infancia a la adolescencia. Australia: Cengage.

Schunk, D. (2012). Teorías del Aprendizaje. Una perspectiva Educativa. Sexta Edición.

México: Pearson Educación. 1997.

Skinner, B. F. (1953). Science and buman behavior. Nueva York: Free Press.

Spearman, C. (1927). The abilities of man. Nueva York: Macmillan.

Sternberg, R. J. (1985). Beyond IQ. A triarchic theory of human intelligence. Cambridge: Cambridge University Press.

Thurstone, L.L. (1938). Primary mental abilities. Chicago: University of Chicago Press.

Vygotski, L. 1979. Desarrollo de procesos psicológicos superiores. Barcelona: Crítica-Estudios y Ensayos.

Watson, J.B. (1913). Psychology as the behaviorist views it. Psychological Review, 20, 158-177. 